\title{
IGDT-based Robust Power Dispatch for Active Distribution Network Considering Demand Response
}

\author{
Huihua Zhuang ${ }^{1}$, Huimin Zhuang ${ }^{2 *}$, \\ ${ }^{1}$ Locomotive and rolling stock Department, Kunming Railway Vocational and Technical College, Kunming, China \\ ${ }^{2}$ Department of Control Engineering, Chengdu University of Information Technology, Chengdu, China
}

\begin{abstract}
Considering increasing uncertain renewable energy sources (RES) and flexible loads in active distribution network $(\mathrm{ADN})$, this study proposes a novel optimal model for robust hourly energy scheduling of ADN. Firstly, a deterministic optimal dispatching model is formulated, which aims at minimizing the total operation cost of distribution network; Secondly, the information gap decision theory (IGDT) is employed to handle uncertainties of RES generation. One of the features of the proposed model is to take into account the impact of demand response of flexible loads and energy storage system (ESS) as the effective tools to reduce unintended costs due to uncertainty of RESs. Also, the uncertainty of RESs is handled in a way that maximum tolerable uncertainty is achieved for a given worsening of total operation cost. The model is formulated as a mixed integer nonlinear optimization problem and solved in the genetic algorithm. Numerical simulation on the IEEE 33-bus system has been performed. Comparisons with two types of probabilistic techniques demonstrate the effectiveness and benefits of the proposal.
\end{abstract}

\section{INTRODUCTION}

With the increasing number of the DGs, the traditional distribution network is going to be gradually transformed into the active distribution network (ADN), which is capable of coordinating DGs, energy storage systems (ESS), demand-side response to keep the distribution network operate in security and economy [1].

However, the active power output of distributed renewable generators (DRGs), such as photovoltaic (PV) and wind power generation (WT) units, may fluctuate with the changing weather conditions and is hard to predict accurately. A deterministic power dispatch model, ignoring DG output uncertainty, fails to guarantee system security and cost optimality in practice because it makes decisions based on only one possible scenario. Therefore, uncertainties in the DRG output should be taken into account for the power dispatch models to ensure system security in all possible scenarios while providing statistically optimal active power allocation schedule.

To cope with the uncertainties brought by DRGs in power dispatch, a variety of approaches with different stochastic variables and constraints embedded have been employed extensively, such as stochastic optimization (SO) and robust optimization (RO). In SO, random variables with a known probability distribution are generally used to account for the output of DRGs. A chance-constrained economic dispatch problem was solved in [2], where the wind power forecast error was addressed as a multivariable probability distribution model. In [3], a scenario generation and reduction algorithm based on particle swarm optimization has been used to model the uncertainties of wind generation. Reference [4] has developed a scenario-based stochastic optimal power flow (OPF) model and solved it in a decentralized scheme, where a set of scenarios are used to describe the uncertainties. Compared with SO, RO has the following upsides: more computationally tractable and easier to obtain empirical or predictive knowledge required by the uncertainty set of DRG output. However, RO tends to make more conservative dispatch decisions, and being robust to unnecessary situations could even lead to infeasibilities on some occasion. In [5], the worst-case transaction cost caused by the uncertainty of DRGs is treated as the optimal objective to model the power scheduling, which is solved in a distributed approach.

Recently, IGDT has shown a great promise in power system applications to handle uncertainty since it neither needs the probability density function nor the variation interval of uncertain parameters unlike $\mathrm{SO}, \mathrm{RO}$ and DRO [6]. In [7-8], the IGDT is applied to deal with the uncertainties associated with RES output or load demand for unit commitment or energy management of power system. Alison et al [9] focus on an IGDT based threephase optimal power flow to deal with the problem of LV distribution network operation under uncertainty.

This paper proposes a new robust optimization model for the economic dispatch of ADN with high-penetration RESs and flexible loads based on IGDT. The main contributions of this work include:

\footnotetext{
*Huimin Zhuang: huimin@cuit.edu.cn
} 
(1) A mathematical formulation of day-ahead scheduling for ADN hosting much RES capacity is developed to reduce total operation cost and improve RES penetration simultaneously, along with the application of ESS units and DR of flexible loads.

(2) The IGDT approach is applied to handle the uncertainties of RES output.

(3) An evaluation of effectiveness and robustness in the IGDT-based dispatching scheme is carried out by comparing this proposed technique with probabilistic methods managing the uncertainty, such as scenariobased modelling and the Monte Carlo technique.

The rest of the paper is organized as follows: Section 2 describes two kinds of demand response model. Section 3 presents a deterministic mathematical formulation for the power dispatch in ADN. In Section 4 an IGDT-based robust optimal model is set up considering uncertainty of RESs. In Section5, numeric results on a 33-bus system are shown to illustrate the effectiveness of the proposed model comparing with scenario-based stochastic programming and the Monte Carlo technique. Finally, conclusions are drawn in Section 6.

\section{Demond respond modeling}

The power utility takes the demand response programs to incentivize users to reschedule their energy usage patterns [10]. In terms of trigger mechanism of these programs, demand response is mainly sorted into two branches: Incentive-based Demand Response (IBDR) and Price-based Demand Response (PBDR).

\subsection{IBDR}

According to the incentive means, IBDR can be divided into direct load control (DLC), interruptible load (IL), emergency demand reduction (EDR) and so on. This paper focuses on IL incentive mode. In this model, the power enterprise signs a contract with the power users who participate in the action to stipulate the reserved capacity cost and interrupted energy cost, which consist the response cost of IL as follows:

$$
C^{\mathrm{IL}}=A \cdot d^{\mathrm{IL}}+\sum_{t=1}^{T} B \cdot e_{t}^{\mathrm{IL}} \cdot \Delta t
$$

Where $d^{\mathrm{IL}}, e_{t}^{\mathrm{IL}}$ are the reserved interruptible capacity (MW) and actual curtailed capacity(MW) respectively, $\mathrm{A}$ and $\mathrm{B}(\$ / \mathrm{MW})$ are the unit capacity compensation price for $d^{\mathrm{IL}}$ and $e_{t}^{\mathrm{IL}}$ respectively, $\Delta t$ is the response cycle.

The actual interrupted capacity should be within the allowable range of the reserved interruptible capacity. And the constraint about IL is expressed as follow:

$$
z_{t} \cdot D^{\mathrm{IL}, \min } \leq e_{t}^{\mathrm{IL}} \leq z_{t} \cdot D^{\mathrm{IL}, \max }
$$

Where $z_{t}$ is a Boolean state variable, depicting whether users are willing to be interrupted or curtailed; $D^{\mathrm{IL} \text {,min }}$ and $D^{\mathrm{IL}, \max }$ are the lower and upper limits of interrupted capacity respectively.

\subsection{PBDR}

PBDR refers to the load demand change according to the variance of electricity price so as to reduce total power consumption cost. The load demand response Dt of users participating in response is modeled by price-elastic demand function as follow[10]:

$$
D_{t}=P_{t}^{\mathrm{D} 0} \cdot\left(\varepsilon_{t t} \cdot \frac{\rho_{t}-\rho_{t}^{0}}{\rho_{t}^{0}}+\sum_{\substack{h=1 \\ h \neq t}}^{T} \varepsilon_{t h} \cdot \frac{\rho_{h}-\rho_{h}^{0}}{\rho_{h}^{0}}\right)
$$

Where $t, h$ represent two different time slots, $\varepsilon t t$ is self-elasticity factor, and $\varepsilon$ th is cross-time elasticity factor of time slot $h$ versus $t, \rho t 0$ and $\rho t$ are the reference price and the price after altering price in slot $t$ respectively, PtD0 denotes initial load demand in slot $t$.

Constraints for IBDR are listed below, including energy balance constraints (4) and capacity ones (5) of $\mathrm{DR}$, as well as price constraints (6).

$$
\begin{gathered}
\sum_{t=1}^{T} D_{t}=0 \\
P_{t, \text { min }}^{\mathrm{D}}-P_{t}^{\mathrm{D} 0} \leq D_{t} \leq P_{t, \text { max }}^{\mathrm{D}}-P_{t}^{\mathrm{D} 0} \\
\rho_{t}^{\text {min }} \leq \rho_{t} \leq \rho_{t}^{\text {max }}
\end{gathered}
$$

Where Pt,maxD and Pt,minD are limits of priceelastic load demand; $\rho$ tmax and $\rho$ tmin are limits of electricity price.

\section{Deterministic optimal model}

In this section, the optimization model for minimizing the total operation cost of ADN is only carried out under the base case (BC) where RES output and load demand are equal to their forecasted value.

This objective function is established as follows:

$$
\begin{gathered}
F_{0}=\min \left(C_{1}+C_{2}+C_{3}+\rho^{\mathrm{Rc}} \zeta_{i, t} P_{i, t}^{\mathrm{R} 0}\right) \\
C_{1}=C_{11}+C_{12}=\sum_{t=1}^{T} \rho_{t}^{0}\left(P_{t}^{\mathrm{F}}+\sum_{i j \in \boldsymbol{\Omega}_{\mathrm{b}}} r_{i j} I_{i j, t}^{2}\right) \Delta t \\
C_{2}=C_{21}+C_{22}=\sum_{t=1}^{T} \sum_{i=1}^{n}\left[\left(a_{i} P_{i, t}^{\mathrm{G}}+b_{i}\right)+\left(\rho_{i}^{\mathrm{c}} P_{i, t}^{\mathrm{Ec}}+\rho_{i}^{\mathrm{d}} P_{i, t}^{\mathrm{Ed}}\right)\right] \Delta t \\
C_{3}=\sum_{i=1}^{n}\left[C_{i}^{\mathrm{L}}+\sum_{t=1}^{T}\left(P_{i, t}^{\mathrm{D} 0}\left(\rho_{t}^{0}-\rho_{t}\right)-D_{i, t} \rho_{t}\right)\right]
\end{gathered}
$$

Where C11 represents the cost of purchasing electricity from the main network, $\mathrm{C} 12$ is the power-loss cost of $\mathrm{ADN}, \mathrm{C} 2$ denotes the generation cost of the dispatchable distributed energy resources, including gas turbines and ESSs, C3 is the cost for scheduling DRs involving IBDR and PBDR; Pi,tR0 and $\zeta$ i,t and are respectively the forecast output of RES the curtailed power rate of RES located the node $i$ at the period $t, \rho R c$ is the curtailing-output cost coefficient; $\mathrm{T}, \Delta \mathrm{t}$ are respectively the number of scheduling time slots and the time interval; PtM is the active power injected into the $\mathrm{ADN}$ from the main network at the period $\mathrm{t} ; \mathrm{n}$ is the number of nodes in ADN, $\boldsymbol{\Omega}_{\mathrm{b}}$ is the set of buanches, rij is the resistance of branch $\mathrm{ij}$, Iij,t is the current flowing through branch $\mathrm{ij}$ at the period $\mathrm{t}$; $\mathrm{Pi}, \mathrm{tG}$ is the output of the gas turbine located the node $\mathrm{i}$ and ai,bi are 
coefficients of generation cost; $\mathrm{Pi}, \mathrm{tEc}$ and $\mathrm{Pi}, \mathrm{tEd}$ are respectively the charging power and discharging power of the ESD located the node $i$ at the period $t$, and pic and pid are respectively its charging and discharging cost coefficients.

Here, the three-level (on-peak, mid-peak, off-peak) time blocks-based ToU pricing is employed for $\mathrm{\rho t} 0$.

Constraints are stated as follows:

1) Power flow constraints

The equations representing the steady-state operation of a radial DN are shown in (8), which is frequently used in the load flow sweep method:

$$
\begin{gathered}
P_{j, t}=\sum_{k \in \delta(j)} P_{j k, t}-\sum_{i \in \pi(j)}\left(P_{i j, t}-\tilde{I}_{i j, t} r_{i j}\right), \vee j \in \boldsymbol{\Omega}_{\mathrm{n}} \\
Q_{j, t}=\sum_{k \in \delta(j)} Q_{j k, t}-\sum_{i \in \pi(j)}\left(Q_{i j, t}-\tilde{I}_{i j, t} x_{i j}\right), \vee j \in \boldsymbol{\Omega}_{\mathrm{n}} \\
\tilde{V}_{j, t}=\tilde{V}_{i, t}-2\left(P_{i j, t} r_{i j}+Q_{i j, t} x_{i j}\right)+\tilde{I}_{i j, t}\left(r_{i j, t}^{2}+x_{i j, t}^{2}\right), \vee i j \in \boldsymbol{\Omega}_{\mathrm{b}} \\
P_{i j, t}^{2}+Q_{i j, t}^{2}=\tilde{V}_{i, t} \tilde{I}_{i j, t}, \vee i j \in \boldsymbol{\Omega}_{\mathrm{b}}
\end{gathered}
$$

Where $\tilde{I}_{i j, t}=I_{i j, t}^{2}, \tilde{V}_{i, t}=V_{i, t}^{2}$. $\boldsymbol{\Omega}_{\mathrm{n}}$ is the set of nodes, $P_{i j, t}$, $Q_{i j, t}$ are respectively active power flow and reactive power flow of branch $i j$ at the period $t, \delta(j)$ is the set of branch terminal nodes with $\mathrm{j}$ as the head node, $\pi(\mathrm{j})$ is the set of branch head nodes with $\mathrm{j}$ as the terminal node, $x i j$ is the reactance of branch $\mathrm{ij}, \mathrm{Vi}, \mathrm{t}, \mathrm{Vj}, \mathrm{t}$ are respectively the voltage amplitude of the node $\mathrm{i}$ and $\mathrm{j}$ at the period $\mathrm{t}, \mathrm{Pj}, \mathrm{t}$ and $\mathrm{Qj}, \mathrm{t}$ are respectively active power and reactive power injected into the node $\mathrm{j}$ at $\mathrm{t}$, which are expressed as (10):

$$
\begin{aligned}
& P_{j, t}=P_{j, t}^{\mathrm{G}}+\left(1-\zeta_{j, t}\right) P_{j, t}^{\mathrm{R} 0}+s_{j, t}^{\mathrm{d}} P_{j, t}^{\mathrm{Ed}}-s_{j, t}^{\mathrm{c}} P_{j, t}^{\mathrm{Ec}}-P_{j, t}^{\mathrm{L}} \\
& Q_{j, t}=\lambda_{j}^{\mathrm{G}} P_{j, t}^{\mathrm{G}}+Q_{j, t}^{\mathrm{cp}}-\lambda_{j}^{\mathrm{L}} P_{j, t}^{\mathrm{L}} \\
& P_{j, t}^{\mathrm{L}}=L_{j, t}+\left(P_{j, t}^{\mathrm{IL}}-e_{j, t}^{\mathrm{IL}}\right)+\left(P_{j, t}^{\mathrm{D} 0}+D_{j, t}\right)
\end{aligned}
$$

Where $\mathrm{Pj}, \mathrm{tL}$ is the active power of load located at the node $\mathrm{j}$ at the period $\mathrm{t}$, Qj,tcp is the reactive power injected by the reactive power compensator located at the node $\mathrm{j}$ at the period $\mathrm{t}, \lambda \mathrm{jG}$ and $\lambda \mathrm{jL}$ are respectively the tangent of power-factor angel of gas turbine and load located at the node $\mathrm{j}$, WT operates in unit power factor in the paper. $s_{i, t}^{\mathrm{c}}, s_{i, t}^{\mathrm{d}}$ are bool variables: ${ }_{i, t}^{\mathrm{c}}=1$ means the ESS is extracting power from the ADN and the ESS is injecting power into the $\mathrm{ADN}$ if $s_{i, t}^{\mathrm{d}}=1, \mathrm{Lj}, \mathrm{t}$ and $\mathrm{Pj}, \mathrm{tIL}$ are the power of non-responsible loads and the original power of IL at the node $\mathrm{j}$ at the period $\mathrm{t}$, respectively.

2) Operational constrains of the network

In order to ensure the safe operation of distribution network, the voltage of each node and the current of each branch should not exceed their limits as follows:

$$
\begin{aligned}
& V_{\min }^{2} \leq \tilde{V}_{i, t} \leq V_{\max }^{2} \\
& \tilde{I}_{i j, t} \leq I_{i j, \max }^{2}
\end{aligned}
$$

Where Vmax, Vmin are respectively the upper and lower limits of voltage, Iij,max is the maximum current in the branch ij.

3) Output constrains of the main network

$$
\begin{aligned}
& P_{\min }^{\mathrm{M}} \leq P_{t}^{\mathrm{M}} \leq P_{\max }^{\mathrm{M}} \\
& Q_{\min }^{\mathrm{M}} \leq Q_{t}^{\mathrm{M}} \leq Q_{\max }^{\mathrm{M}}
\end{aligned}
$$

Where PMmin , QMmin, PMmax , QMmax are the limited active power and reactive power injected from the main network respectively.

4) Operational constrain of gas turbines

The generation and ramping limitations are included:

$$
\begin{aligned}
& P_{i, \text { min }}^{\mathrm{G}} \leq P_{i, t}^{\mathrm{G}} \leq P_{i, \text { max }}^{\mathrm{G}} \\
& -r_{i}^{\mathrm{d}} \Delta t \leq P_{i, t}^{\mathrm{G}}-P_{i, t-1}^{\mathrm{G}} \leq r_{i}^{\mathrm{u}} \Delta t
\end{aligned}
$$

Where $P_{i, \text { max }}^{\mathrm{G}}, P_{i, \text { min }}^{\mathrm{G}}, r_{i}^{\mathrm{u}}, r_{i}^{\mathrm{d}}$ are respectively maximum and minimum active output and ramp-up rate and rampdown rate of the gas turbine located at the node $i$.

5) Operational stae constraint of ESSs

For the ESS at node i, its operation state should satisfy this constraint below at the period $t$ :

$$
\begin{gathered}
E_{i, t+1}=E_{i, t}+\left(\eta_{\mathrm{c}} P_{i, t}^{\mathrm{Ec}}-P_{i, t}^{\mathrm{Ed}} / \eta_{\mathrm{d}}\right) \Delta t \\
0 \leq P_{i, t}^{\mathrm{Ec}} \leq s_{i, t}^{\mathrm{c}} P_{i, \max }^{\mathrm{Ec}} \\
0 \leq P_{i, t}^{\mathrm{Ed}} \leq s_{i, t}^{\mathrm{d}} P_{i, \max }^{\mathrm{Ed}} \\
E_{i, \min } \leq E_{i, t} \leq E_{i, \max } \\
s_{i, t}^{\mathrm{c}}+s_{i, t}^{\mathrm{d}} \leq 1
\end{gathered}
$$

Where Ei,t denotes capacity of energy storage, $\eta c$ and $\eta \mathrm{d}$ are efficiency of power extraction and injection respectively, Pi,maxEc, Pi,maxEd, Ei,max、Ei,min are respectively maximum extraction power, maximum injection power upper and lower limits of capacity.

6) Constraint of Curtailed WT output

$$
0 \leq \zeta_{j, t} \leq 1
$$

Besides, the constraints about DR shown as (2), (5) and (6) are also included.

In summary, the optimal model above of $\mathrm{BC}$ is formulated as below in the matrix form:

$$
\begin{array}{ll} 
& \min F_{0}(\boldsymbol{x}, \bar{\psi}) \\
\text { s.t. } & G(\boldsymbol{x}, \bar{\psi})=0 \\
& H(\boldsymbol{x}, \bar{\psi}) \leq 0
\end{array}
$$

Where $\mathrm{x}$ represents decision-variable vector; $\bar{\psi}$ denotes the forecasted values of RES generation, which is composed of Pi,tR0; the formula (18) consists of (8) to (10) and (14); (19) consists of (11) to (13), (15) and (16).

\section{IGDT-based robust optimal model}

To cope with the uncertainty of RES power generation, an IGDT-based optimization approach is employed, which can effectively optimize the uncertainty index in problems suffering from severe lack of information to make well-qualified decision [7]. This method has two immune models to handle uncertainties: RS IGDT-based model and RA IGDT-based model which is adopted here due to its robustness. Uncertain values are characterized with several models. This paper selects the envelope bound uncertainty model shown below:

$$
U(\bar{\psi}, \gamma)=\{\psi:|\psi-\bar{\psi}| \leq \gamma|\bar{\psi}|\}
$$


Where $\psi, \gamma$ represent input uncertain parameters, i.e. $P_{i, t}^{\mathrm{R}}$ and uncertainty radius, respectively.

When the RA strategy is adopted, it will be sure that the objective function value should not exceed the preset threshold as far as the uncertain parameter alters within the tolerable uncertainty radius. So the mathematical equations describing RA strategy are expressed as:

$$
\begin{gathered}
\gamma_{\mathrm{c}}=\max _{\boldsymbol{x}} \gamma \\
\text { s.t. } \quad \max _{\psi} F(\boldsymbol{x}, \psi) \leq F_{0}(1+\sigma) \\
G(\boldsymbol{x}, \psi)=0 \\
H(\boldsymbol{x}, \psi) \leq 0 \\
0 \leq \gamma \leq 1 \\
\psi \in U(\bar{\psi}, \gamma)
\end{gathered}
$$

Where $\sigma$ is the conservativeness factor.

The RA IGDT-based scheduling model above is a bi-level problem. To solve the IGDT-based optimal model, the bi-level formulation should be reformulated as a single level problem. The maximal operation cost is seen to happen at the minimum RES generation as shown in (27).

$$
P_{j, t}^{\mathrm{R}}=(1-\gamma) P_{j, t}^{\mathrm{R} 0}
$$

Then the RA IGDT-based optimization model can be formulated by:

$$
\begin{array}{ll} 
& \gamma_{\mathrm{c}}=\max _{\boldsymbol{x}, \psi} \gamma \\
\text { s.t. } & F(\boldsymbol{x}, \psi) \leq F_{0}(1+\sigma)
\end{array}
$$$$
\text { (23) to }(25),(27)
$$

The proposed optimal model above is a mix integer nonlinear programing (MINLP) one and solved by the genetic algorithm with good global convergence.

\section{Case study}

In this section, the approach put forward in this paper has been applied for a modified IEEE 33-bus test system to illustrate the performance of the proposed model and algorithm. The simulation is performed on a $2.9 \mathrm{GHz}$ personal computer with 16 GB RAM. And the algorithm is implemented in MATLAB R2016a and programmed through YALMIP.

\subsection{Test system data}

A modified IEEE 33-bus distribution network plotted in Fig. 2 is employed for verifying the proposed method. Two PV generators and two WT ones are respectively installed at buses \#6, \#16, \#24, \#31. The nominal capacity of each PV and WT is $0.5 \mathrm{MWand} 0.8 \mathrm{MW}$ respectively. A micro gas turbine (MT) with the power factor being 0.95 is installed at bus \#20. Two ESSs with $\eta \mathrm{c}$ and $\eta \mathrm{d}$ being both 0.9 are installed at bus \#17 and \#29 respectively. loads at bus \#25 and \#32 are both PBDR and ones at bus $\# 8$ and $\# 30$ are both ILs. Other parameters are seen in [5]. The total peak load of the system is $5.084 \mathrm{MW}$. The forecasted values of load demand along with power output of PV and WT for a typical $24 \mathrm{~h}$ time period are depicted in figure 2 . It is worth to note that the values for demand and power generations are respected to the corresponding peak values. The energy market prices for the considered period are given in Table 1. The safe range of voltage is from $0.95 \mathrm{pu}$ to $1.05 \mathrm{Pu}$, and the upper and lower limits of line current are $4 \mathrm{pu}$ and $-4 \mathrm{pu}$, respectively. The maximum power injected by the main network is 5MW.

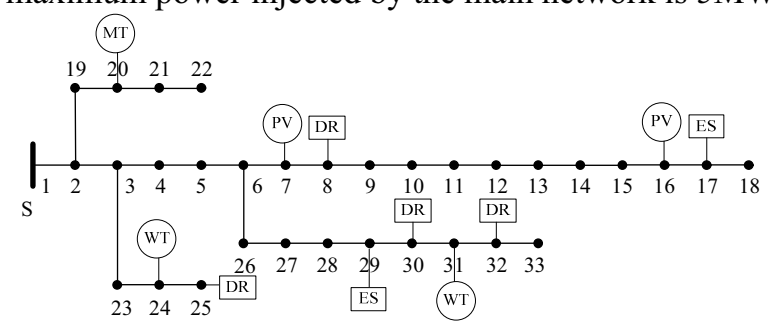

igure 1. Modified IEEE33-bus distribution network

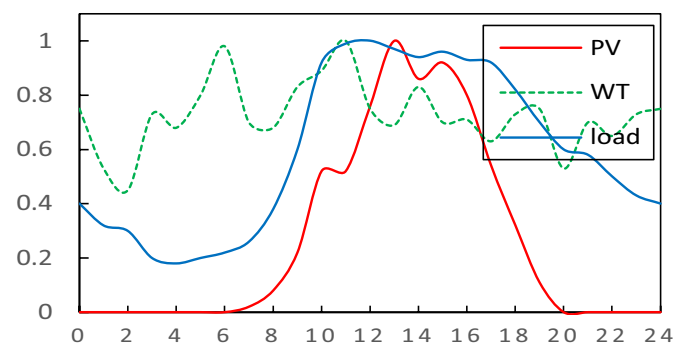

Figure 2. Prediction value of RESs and load Table 1. Setting TOU price.

\begin{tabular}{|c|c|}
\hline Time intervals & $\begin{array}{c}\text { Electricity price } \\
\boldsymbol{\rho}_{t}^{\mathbf{0}} \text { (\$/MWh) }\end{array}$ \\
\hline $\begin{array}{c}\text { 10:00-15:00, 18:00-21:00 } \\
\text { 7:00-10:00,15:00-18:00, } \\
21: 00-23: 00\end{array}$ & 194.41 \\
\hline $23: 00-7: 00$ & 122.35 \\
\hline
\end{tabular}

\section{$5.2 \mathrm{BC}$ condition}

In this case, the load demand along with the output of PV and WT all equal their corresponding forecasted values, i.e. $\gamma=0$. The proposed method in this paper is applied to acquire the optimal power dispatch scheme, which yields optimal results: the total operation cost is $6550.38 \$$ and the output of each controllable resource is depicted in Figure 3. Figure 4 shows the charged (negative) or discharged (positive) power of each ESS and the energy consumption of each PBDR load. The total curtailed wind-power rate is about $4.56 \%$ during 2:00 6:00 for satisfying safety constraint of the network. 


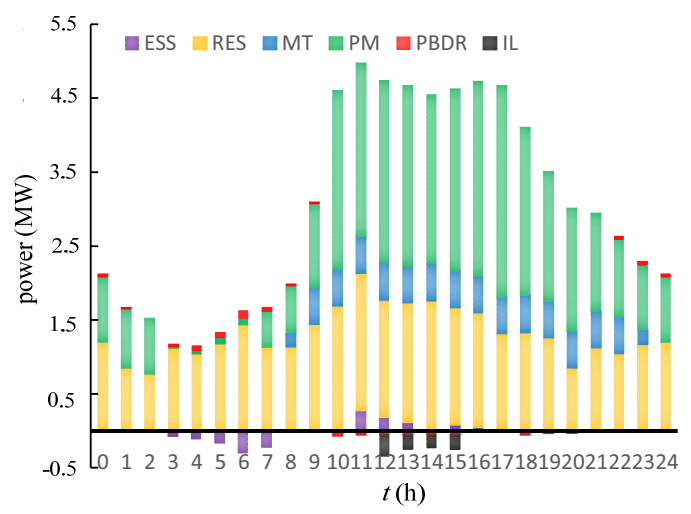

Figure 3. optimal scheduling under BC

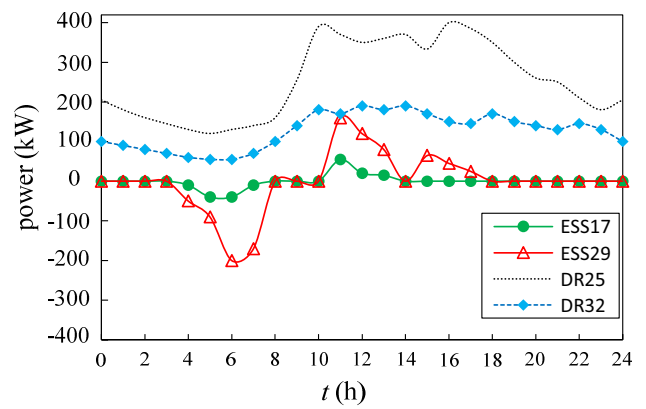

Figure 4. optimal power of ESS and PBDR under BC

It is observed from Figure 3 and Figure4 that ESSs are charged from 03:00 to 07:00 during the off-peak period, and discharged to ADN from 11:00 to 16:00 in the peak hour interval, IL programme is activated from 12:00 to $15: 00$, and the demand of PBDR decreases in the intervals where the electricity price is high and is shifted to the low-energy-price hours, which indicates DR- loads and ESSs both play an important role in peak shaving and off-peak filling.

\subsection{RA condition}

The DN operator aims to raise the robustness of the objective function in this case. Hence, the maximum uncertainty radius is expected to be fund to ensure that the total cost will not deviate from its base case value more than a certain level which is defined by conservativeness factor $\sigma$ in (22). In case of $\sigma$ being $8 \%$, the maximum uncertainty radius $\gamma c$ is 32.85 , and the energy served by ESS and energy consumption during 24 hours are depicted in Fig.5. It is seen that in contrast with the base case, ESSs absorb more energy from the network during off-peak intervals and inject more energy into the network during peak hours, meanwhile PBDRs shift more energy-consumption from peak hours to offpeak ones by varying energy price in real time.

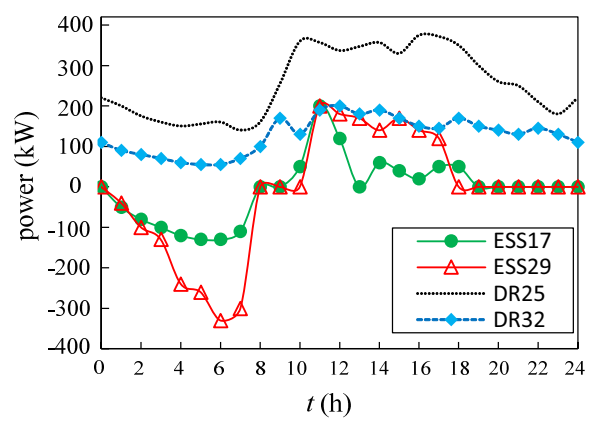

Figure 5. optimal power of ESS and PBDR in case of $\sigma=8 \%$

While this parameter $\sigma$ varies from 0.02 to 0.1 with the step of 0.02 , the variables including the maximum uncertainty radius $\gamma_{\mathrm{c}}$, the objective function $\mathrm{F}$ and the total curtailed RES power ratio $\zeta$ are provided in the table 2. It is observed from Table 4 that with the increasement of $\sigma$, the tolerable uncertainty of net load is rising constantly until $\sigma$ equals $18 \%$ and then keep constant. It illustrated that the energy difference caused by the uncertainty can be supplied by controllable resources such as ESSs and DRs when the uncertainty is lower than $18 \%$, but while $\sigma$ is beyond $18 \%$, the regulative capacity of various controllable resources will be not enough to balance this energy difference. That is to say, the uncertainty this system is tolerant of should not exceed $54.02 \%$ for sake of safe operation. On the other hand, the curtailed power of RES varies slightly before $\sigma$ is increased to $10 \%$ and then rises substantially.

Table 2. Optimal results in case of different $\sigma$.

\begin{tabular}{|c|c|c|c|}
\hline$\sigma(\%)$ & $\gamma_{c}(\%)$ & $\mathbf{F}(\mathbf{})$ & $\zeta(\%)$ \\
\hline 2 & 11.37 & 6591.23 & 4.56 \\
\hline 4 & 19.19 & 6741.79 & 4.58 \\
\hline 6 & 25.54 & 6883.05 & 4.63 \\
\hline 8 & 32.85 & 7053.81 & 4.75 \\
\hline 10 & 43.42 & 7200.18 & 8.04 \\
\hline 12 & 48.27 & 7305.69 & 10.75 \\
\hline 14 & 49.33 & 7386.82 & 12.32 \\
\hline 16 & 52.97 & 7517.68 & 15.93 \\
\hline 20 & 54.02 & 7821.95 & 16.08 \\
\hline
\end{tabular}

\subsection{Comparison between RA strategy and other uncertainty handling methods}

The propsed IGDT-based RA strategy is compared with two probilistic approahes: scenario-based stochastic programming(SBSP) technique and Monte Carlo 
simulations (MCS). To employ SBSP method, it is assumed that the uncertain RES generation follows a multivariate normal distribution with 0 as the mean value and 0.3 times of the predicted value as the variance. 10000 error scene data are randomly generated based on the predicted scene data, then 10 typical scenarios are generated by the k-means cluster algorithm. In addition, 1000 random samples of RES output are generated uniformly in the interval of [0.7Pi,tR0 ,1.3 Pi,tR0] for using MCS. The results solved by SBSP technique and MCS are summarized in table 3 in comparison with RA strategy. It can be observed from this table that the CPU time in the case of IGDT-based RA is evidently less than other two approaches, and the expected cost in use of SBSP is higher than both RA and MCS techniques.

\section{Conclusion}

This paper proposes a robust optimal framework to develop a day-ahead scheduling scheme considering the uncertainty of RES generation for the ADN with high penetration of RESs.

Table 3. Comparison of different algorithms.

\begin{tabular}{|c|c|c|c|}
\hline Method & CPU-time (s) & $\begin{array}{c}\text { Expected } \\
\text { cost } \mathbf{( \$ )}\end{array}$ & $\begin{array}{c}\text { Standard } \\
\text { deviation (\$) }\end{array}$ \\
\hline IGDT & 306 & 7053.81 & - \\
\hline SBSP & 1473 & 7102.88 & 69.64 \\
\hline MCS & 14269 & 7039.20 & 22.17 \\
\hline
\end{tabular}

The IGDT technique is employed to handle the uncertainty. ESS and PBDR being of great flexibility are used to restrain the RES output fluctuation for raising RES penetration in ADN. This dispatching scheme aims at minimizing the total operation cost of $\mathrm{ADN}$ by controlling the power procured from the main network, output of dispatchable DGs (DDG) and the reserved interruptible capacity of IL, ESS power and the real-time electricity price. Then the genetic algorithm is applied to solve this optimal model.The comparison with the traditional stochastic and robust approaches on IEEE 33bus test system indicates that the proposed model can achieve better optimal solution and computational performance than traditional methods.

\section{References}

1. A. W. Bizuayehu, A. A. S. d. 1. Nieta, J. Contreras et al. Impacts of Stochastic Wind Power and Storage Participation on Economic Dispatch in Distribution Systems. IEEE Transactions on Sustainable Energy, vol. 7, no. 3, pp. 1336-1345, 2016.

2. Z. S. Zhang, Y. Z. Sun, D. W. Gao et al. A Versatile Probability Distribution Model for Wind Power Forecast Errors and Its Application in Economic Dispatch. IEEE Transactions on Power Systems, vol. 28, no. 3, pp. 3114-3125, 2013.
3. W. Zheng, W. Wu, B. Zhang et al.. Fully distributed multi-area economic dispatch method for active distribution networks. IET Generation, Transmission \& Distribution, vol. 9, no. 12, pp. 1341-1351, 2015.

4. M. Bazrafshan, and N. Gatsis. Decentralized Stochastic Optimal Power Flow in Radial Networks With Distributed Generation. IEEE Transactions on Smart Grid, vol. 8, no. 2, pp. 787-801, 2017.

5. LIANG Junwen, LIN Shunjiang, LIU Mingbo, etc. Distributed Robust Optimal Dispatch in Active Distribution Networks. Power System Technology, vol.43, no.4, pp. 1336-1344, 2019.

6. Ke, D.P.; Shen, F.F.; Chung, C.Y.; et al. Application of information gap decision theory to the design of robust wide-area power system stabilizers considering uncertainties of wind power. IEEE Trans. Sustain. Energy, vol. 9, no. 9, pp. 1-10, 2017.

7. Rabiee A.; Soroudi A.; Keane A. Information gap decision theory based opf with hvdc connected wind farms. IEEE Trans Power Syst, vol. 3, no. 6, pp.3396-3406, 2015.

8. Rabiee A.; Nikkhah S.; Soroudi A. Information gap decision theory to deal with long-term wind energy planning considering voltage stability. Energy, vol. 14,no.7, pp. 451-463, 2018.

9. O’Connell A.; Soroudi A.; Keane A. Distribution network operation under uncertainty using information gap decision theory. IEEE Trans. Smart Grid, vol. 9, no. 3, pp.1848-1858., 2018.

10. Moghaddam M.P.; Abdollahi A.; Rashidinejad M. Flexible demand response programs modeling in competitive electricity markets. Appl. Energy, vol. 88, pp. 3257-3269, 2011. 